\title{
BMJ Open The role of cognitive function in the relationship between age and health literacy: a cross-sectional analysis of older adults in Chicago, USA
}

\author{
Lindsay C Kobayashi, ${ }^{1}$ Samuel G Smith, ${ }^{1,2}$ Rachel O'Conor, ${ }^{3}$ Laura M Curtis, ${ }^{3}$
} Denise Park, ${ }^{4}$ Christian von Wagner, ${ }^{1}$ Ian J Deary, ${ }^{5}$ Michael S Wolf ${ }^{3,6}$

To cite: Kobayashi LC, Smith SG, O'Conor R, et al. The role of cognitive function in the relationship between age and health literacy: a cross-sectional analysis of older adults in Chicago, USA. BMJ Open 2015;5:e007222. doi:10.1136/bmjopen-2014007222

- Prepublication history for this paper is available online. To view these files please visit the journal online (http://dx.doi.org/10.1136/ bmjopen-2014-007222).

Received 16 November 2014 Revised 19 February 2015 Accepted 20 March 2015

CrossMark

For numbered affiliations see end of article.

Correspondence to

Michael S Wolf;

mswolf@northwestern.edu

\section{ABSTRACT}

Objectives: To investigate how 3 measures of health literacy correlate with age and the explanatory roles of fluid and crystallised cognitive abilities in these relationships among older adults.

Design: Cross-sectional baseline analysis of the 'LitCog' cohort study.

Setting: 1 academic internal medicine clinic and 5 federally qualified health centres in Chicago, USA.

Participants: English-speaking adults $(n=828)$ aged 55-74 years, recruited from August 2008 through October 2011.

Outcome measures: Health literacy was measured by the Test of Functional Health Literacy in Adults (TOFHLA) and the Newest Vital Sign (NVS), both of which assess reading comprehension and numeracy in health contexts, and by the Rapid Estimate of Adult Literacy in Medicine (REALM), which assesses medical vocabulary. Fluid cognitive ability was assessed through the cognitive domains of processing speed, inductive reasoning, and working, prospective and long-term memories, and crystallised cognitive ability through the verbal ability domain.

Results: TOFHLA and NVS scores were lower at ages 70-74 years compared with all other age groups $(p<0.05$ for both tests). The inverse association between age and TOFHLA score was attenuated from $\beta=-0.39(95 \% \mathrm{Cl}-0.55$ to -0.22$)$ to $\beta=-0.06(95 \%$ $\mathrm{Cl}-0.20$ to 0.08 ) for ages $70-74$ vs $55-59$ years when fluid cognitive ability was added to the model (85\% attenuation). Similar results were seen with NVS scores (68\% attenuation). REALM scores did not differ by age group ( $p=0.971)$. Crystallised cognitive ability was stable across age groups, and did not influence the relationships between age and TOFHLA or NVS performance.

Conclusions: Health literacy skills show differential patterns of age-related change, which may be explained by cognitive ageing. Researchers should select health literacy tests appropriate for their purposes when assessing the health literacy of older adults. Clinicians should be aware of this issue to ensure that health self-management tasks for older patients have appropriate cognitive and literacy demands.

\section{Strengths and limitations of this study}

- This study simultaneously assesses a range of cognitive abilities and health literacy skills in a sample of older adults.

- This analysis is a cross-sectional design.

- Longitudinal work is needed to establish the threshold at which cognitive ageing may begin to influence various health literacy skills.

\section{INTRODUCTION}

Low health literacy is a major determinant of morbidity and mortality among older adults in the USA. ${ }^{12}$ Among other outcomes, low health literacy is associated with poor chronic disease management, increased risk of hospitalisation, and worse overall health status among older adults. ${ }^{1-4}$ In the 2003 US National Assessment of Adult Literacy, $13 \%$ of American adults aged 50-64 years lacked the basic literacy skills required for health management, rising to $29 \%$ of adults aged 65 years and over. ${ }^{5}$ Despite recent investigations, the specific functional health literacy skills that may be sensitive to age-related decline and their underlying cognitive functions are relatively unknown.

Researchers in the field use several different health literacy measures, each assessing slightly different skill sets. Three commonly used measures are the Test of Functional Health Literacy in Adults (TOFHLA) or its shortened version (S-TOFHLA), the Newest Vital Sign (NVS) and the Rapid Estimate of Adult Literacy in Medicine (REALM). The TOFHLA is a test of reading comprehension and numeracy that uses common health materials such as a health insurance form and X-ray preparation instructions. ${ }^{6}$ The NVS is a reading comprehension and numeracy test using a food nutrition label that requires the reader to respond to information on the label. ${ }^{7}$ The REALM is a 
test of medical vocabulary, which assesses familiarity with medical words and the ability to correctly pronounce them. ${ }^{8}$

Performance on these health literacy tests is correlated with various 'fluid' and 'crystallised' cognitive abilities. Fluid cognitive abilities are those required for active learning and information processing (eg, reasoning, memory, processing speed), and are strongly correlated with performance on the TOFHLA, NVS and similar tests. ${ }^{9-11}$ Crystallised cognitive abilities represent longterm memory or general knowledge (eg, verbal ability or vocabulary), and are strongly correlated with performance on all three of the TOFHLA, REALM and NVS tests. ${ }^{9}$ Longitudinal research shows that fluid cognitive abilities tend to decline beginning in early to midadulthood, while crystallised abilities are stable over time with age. ${ }^{12-14}$

The relationship between the constructs of cognitive ability and health literacy appears to be stable across the life course: in a cohort of Scottish children born in 1936, childhood cognitive ability (age 11 years) was associated with late-life (age $\sim 72$ years) performance on each of the S-TOFHLA, NVS and REALM. ${ }^{15} 16$ In this cohort, relative cognitive change between ages 11 and 70 years predicted late-life S-TOFHLA and NVS scores but not REALM scores. ${ }^{16}$ Performance on the TOFHLA and the NVS may, therefore, exhibit a pattern of age-related decline similar to that of fluid abilities, whereas performance on the REALM may be more stable across age groups due to its relation with crystallised abilities.

The objective of the present analysis was to investigate how health literacy, as assessed by the TOFHLA, REALM and NVS, correlates with age, and the explanatory roles of fluid and crystallised cognitive abilities in these relationships among American adults aged 55-74 years. We hypothesised that fluid cognitive abilities would mostly explain associations between age and health literacy as measured by the TOFHLA and NVS, while crystallised abilities would more likely explain any age-related associations with the REALM.

\section{METHODS}

\section{Study sample}

This analysis used baseline data from the 'Literacy and Cognitive Function in Older Adults' study (hereafter referred to as 'LitCog'). LitCog was established in 2008 to investigate how cognitive ability relates to health literacy skills among older adults. It is a prospective cohort study of English-speaking adults aged 55-74 years, who received care at an academic general internal medicine ambulatory care clinic or at one of five federally qualified health centres in Chicago, Illinois, USA. Electronic health records were used to identify 3176 eligible adults who had had at least two clinic visits within the past 18 months. Of these, 1904 were randomly selected for inclusion in the study. They were notified of the study by mail and were able to opt out at this stage. After screening by telephone, 244 adults were excluded due to cognitive or hearing impairment, limited English proficiency, or lack of affiliation with a clinic physician (ie, less than two recorded visits in the previous 2 years). A total of 794 adults refused, 20 had scheduling conflicts, 14 were deceased, and 4 were duplicate records. The final sample included 828 participants who completed the baseline interview, for a cooperation rate of $51 \%$.

\section{Procedure}

Participants completed two structured interviews, lasting $2 \mathrm{~h}$, conducted 7-10 days apart. On day 1, a trained research assistant guided participants through questions on basic demographic information, comorbidities and the three health literacy measures. On day 2, patients completed a cognitive battery to measure processing speed, working memory, inductive reasoning, long-term memory, prospective memory and verbal ability. With the exception of verbal ability, cognitive tests did not involve reading or numeracy skills. All fluid cognitive domains were assessed using multiple tests to allow a latent trait to be extracted for each. This study was approved by Northwestern University's Institutional Review Board.

\section{Measures}

Health literacy

Health literacy was assessed using the TOFHLA, REALM and NVS. ${ }^{6-8}$ The TOFHLA consists of a 50 -item reading comprehension section that utilises the Cloze procedure, where every fifth to seventh word in a passage is omitted and four multiple choice options for the blank are provided. The TOFHLA also includes a 17 -item numeracy section. Numeracy scores are transformed to match the reading comprehension scores, and the two are summed to give a total out of 100 . Scores are classified as inadequate $(0-59)$, marginal $(60-74)$ or adequate (75-100).

The REALM is a 66-item word-recognition test, where medical words are arranged in order of increasing difficulty, and participants are instructed to read them aloud. Scores are based on the total number of words pronounced correctly, using dictionary pronunciation as assessed by the interviewer. Scores are classified as low (0-44), marginal (45-60) or adequate (61-66).

The NVS is a brief screening tool to determine risk for limited health literacy, where participants read a food nutrition label and respond to six questions about interpreting and acting on the information. Scores are classified as a high likelihood (0-1) or possibility (2-3) of limited health literacy or adequate health literacy (4-6).

Scores on all three tests will be referred to as adequate, as defined above, or as limited $(<75$ on the TOFHLA, $<61$ on the REALM, and $<4$ on the NVS), and will also be analysed continuously as standardised scores.

\section{Cognitive abilities}

Six cognitive domains were assessed through a set of 16 cognitive tests, and latent variables were derived for each 
domain. Briefly, the cognitive domains were processing speed, working memory, inductive reasoning, long-term memory, prospective memory and verbal ability; the specific tests cited here have been described in detail previously. ${ }^{9}$ The former five domains were considered to represent fluid cognitive ability as they are associated with active information processing, whereas the latter domain represented crystallised cognitive ability as it is associated with general background knowledge.

\section{Participant characteristics}

Participant characteristics relevant to this analysis were assessed in the study interview: age (in years: 55-59; 60-64; 65-69; 70-74), gender (male; female), race (African-American; White; other), educational attainment (high school or less; some college or technical school; college graduate; graduate degree), annual income $(<\$ 10000 ; \$ 10000-\$ 24999 ; \$ 25000-\$ 49999$; $\geq \$ 50000$ ), employment status (no work; part-time; fulltime), marital status (married; unmarried) and presence of chronic conditions: arthritis, asthma, bronchitis or emphysema, cancer, coronary heart disease, depression, diabetes, heart failure and hypertension.

\section{Statistical analysis}

Sociodemographics and health conditions of study participants were described, along with the overall proportions with limited health literacy. Raw health literacy scores on each test were transformed into standardised z-scores and compared across age groups using one-way analysis of variance (ANOVA) and Scheffé's multiple comparison test. Univariate imputation sampling methods were used to estimate any missing values ( $n=98)$ on cognitive measures and domain-specific cognitive ability scores were calculated using a latent trait analysis. ${ }^{9}$ One aim of the LitCog study was to investigate the latent cognitive domains that may underlie health literacy skills, hence, latent items were calculated for each cognitive domain (processing speed, working memory, inductive reasoning, long-term memory, prospective memory and verbal ability). ${ }^{9}$ Maximum likelihood estimation was used to estimate single-factor summary scores for general cognitive abilities (fluid and crystallised)..$^{9}$ Mean scores for each of six cognitive domains and overall fluid and crystallised abilities were transformed into standardised z-scores and compared across age groups using one-way ANOVA and Scheffé's multiple comparison test.

Standardised health literacy and cognitive ability scores were plotted by age group to generate a visualisation of trends with age. Multiple linear regression modelling was used to estimate the associations between age and standardised score on each of the TOFHLA, NVS and REALM. All a priori-identified covariates were included in modelling, with standardised fluid and crystallised cognitive ability scores added in a stepwise fashion to determine their separate and combined mediating effects on the relationship between age and health literacy. A post hoc analysis was performed to assess the individual contributions of each of processing speed, working memory, inductive reasoning, prospective memory and long-term memory to the overall mediating effect of fluid cognitive ability on the age-TOFHLA and age-NVS relationships. Health literacy data were missing for 14 participants $(<2 \%)$, and data on one or more cognitive variable were missing and non-imputable for 40 participants $(<5 \%)$, giving an effective sample size of 774. Statistical tests were two sided and conducted at the 95\% confidence level. Analyses were conducted using StataSE V.13.1 (StataCorp, College Station, Texas, USA).

\section{RESULTS}

Participant characteristics are shown in table 1. Two-thirds $(68.4 \%)$ of the sample were women and approximately half were White $(50.6 \%)$. Educational attainment was fairly evenly distributed among the sample (26.4\% had high school or less; $21.7 \%$ had some college or technical school; $20.5 \%$ were college graduates; $31 \%$ had graduate degrees). Over half had an annual income of at least US\$50 $000(53.6 \%)$, and $12 \%$ had an annual income less than US\$10 000. Nearly two-thirds of the sample was not working $(64.6 \%)$. Just under half were married $(44.9 \%)$. Participants had, on average, $1.9 \quad(\mathrm{SD}=1.4) \quad$ chronic health conditions. According to the TOFHLA, $29.1 \%$ of participants had limited health literacy, according to the NVS, 51.6\%, and to the REALM, 24.2\% (table 1).

Mean standardised scores on the TOFHLA and the NVS differed across age groups $(p=0.003$ and 0.0004 , respectively; figure 1). Scheffé's post hoc test showed that TOFHLA and NVS scores were lower at ages $70-74$ years than at all younger age groups $(\mathrm{p}<0.05$ for all $)$. By contrast, REALM scores did not differ by age $(\mathrm{p}=0.971)$. Mean standardised scores for all fluid abilities differed across age groups ( $p<0.05$ for all; figure 1$)$. Scheffé's post hoc test showed that scores for all fluid abilities were lower at ages 70-74 years than at all younger age groups ( $\mathrm{p}<0.05$ for all). By contrast, crystallised (verbal) ability did not differ by age $(\mathrm{p}=0.240$; figure 1$)$.

In multivariable linear regression modelling, standardised TOFHLA and NVS scores were significantly lower in the $70-74$ vs $55-59$ years age group $(\beta=-0.39 ; 95 \%$ CI -0.55 to -0.22 for the TOFHLA, and $\beta=-0.38 ; 95 \%$ CI -0.53 to -0.22 for the NVS; table 2). By contrast, REALM scores were not associated with age. When fluid ability was added to the models, it attenuated the association between age and standardised TOFHLA score by $84.6 \%$ (attenuated $\beta=-0.06 ; 95 \%$ CI -0.20 to 0.08 for the 70-74 vs 55-59 years age group), and attenuated the association between age and standardised NVS score by $68.4 \%$ (attenuated $\beta=-0.12 ; 95 \%$ CI -0.26 to 0.02 for the $70-74$ vs 55-59 years age group). Crystallised ability had no mediating effect on the associations between age and standardised TOFHLA and NVS scores (table 2). The addition of fluid and crystallised abilities together 


\begin{tabular}{|c|c|}
\hline Characteristic & N (\%) \\
\hline \multicolumn{2}{|l|}{ Age (years) } \\
\hline $55-59$ & 239 (30.9) \\
\hline $60-64$ & $250(32.3)$ \\
\hline $65-69$ & 154 (19.9) \\
\hline $70-74$ & $131(16.9)$ \\
\hline \multicolumn{2}{|l|}{ Gender } \\
\hline Male & $245(31.7)$ \\
\hline Female & $529(68.4)$ \\
\hline \multicolumn{2}{|l|}{ Race } \\
\hline African-American & $326(42.4)$ \\
\hline White & $389(50.6)$ \\
\hline Other & $54(7.0)$ \\
\hline \multicolumn{2}{|l|}{ Education } \\
\hline High school or less & $207(26.7)$ \\
\hline Some college or technical school & $168(21.7)$ \\
\hline College graduate & $159(20.5)$ \\
\hline Graduate degree & $240(31.0)$ \\
\hline \multicolumn{2}{|l|}{ Income } \\
\hline$<\$ 10000$ & $87(12.0)$ \\
\hline$\$ 10000-\$ 24999$ & $138(19.1)$ \\
\hline$\$ 25000-\$ 49999$ & $112(15.4)$ \\
\hline$\geq \$ 50000$ & $390(53.6)$ \\
\hline \multicolumn{2}{|l|}{ Employment status } \\
\hline Full-time & $158(20.5)$ \\
\hline Part-time & $114(14.9)$ \\
\hline Not working & $497(64.6)$ \\
\hline \multicolumn{2}{|l|}{ Marital status } \\
\hline Married & $346(44.9)$ \\
\hline Not married & $425(55.1)$ \\
\hline \multicolumn{2}{|l|}{ Chronic conditions } \\
\hline Arthritis & $357(46.8)$ \\
\hline Asthma & $144(18.8)$ \\
\hline Bronchitis or emphysema & 98 (12.9) \\
\hline Cancer & $56(7.2)$ \\
\hline Coronary heart disease & $39(5.2)$ \\
\hline Depression & $152(19.8)$ \\
\hline Diabetes & $119(15.5)$ \\
\hline Heart failure & $36(4.7)$ \\
\hline Hypertension & $458(59.3)$ \\
\hline Mean number of conditions (SD) & $1.9(1.4)$ \\
\hline \multicolumn{2}{|l|}{ Limited health literacy } \\
\hline TOFHLA & $225(29.1)$ \\
\hline NVS & $339(51.6)$ \\
\hline REALM & $187(24.2)$ \\
\hline
\end{tabular}

NVS, Newest Vital Sign; REALM, Rapid Estimate of Adult Literacy in Medicine; TOFHLA, Test of Functional Health Literacy in Adults.

somewhat lessened the attenuation observed with fluid ability only (from $84.6 \%$ to $53.8 \%$ attenuation for the TOHFLA, and from $68.4 \%$ to $52.6 \%$ attenuation for the NVS; table 1). This may be due to the correlation between the two constructs (Spearman's $r=0.76$; $\mathrm{p}<0.0001)$.

Each of processing speed, working memory, inductive reasoning, prospective memory and long-term memory mediated the age-TOFHLA performance relationship to some degree (table 3). However, it appeared that processing speed was the strongest mediator and that prospective and long-term memory minimally contributed to mediation (table 3). Together, the contributions of these cognitive abilities led to the overall degree of mediation shown by the 'fluid cognitive ability' construct in table 2. With respect to the NVS, the individual cognitive abilities did not explain age differences in performance to the same degree as observed with the TOFHLA (table 3). However, the incremental contributions of the individual abilities to the overall mediating effect of 'fluid cognitive ability' mostly explained the age-NVS performance relationship, as shown in table 2.

\section{DISCUSSION}

Among a sample of English-speaking older American adults, performance on the TOFHLA and the NVS appeared to be lowest in the oldest age group (70-74 years), with a threshold rather than a graded trend across age groups. Performance on tests of fluid cognitive abilities demonstrated a more graded decline with increasing age, with the poorest abilities seen in the oldest age group such as with the TOFHLA and the NVS. As with performance on the REALM, crystallised verbal ability remained constant with age. As fluid abilities nearly fully attenuated the association between both the TOFHLA and the NVS with age, it can be postulated that these two constructs have significant overlap with regard to their roles during ageing. In support of our hypotheses, neither crystallised cognitive ability nor performance on the REALM had any relationship with age.

This study supports the findings of a recent systematic review showing that performance on the REALM is more stable with increasing age than is performance on the TOFHLA and NVS. ${ }^{17}$ Two studies of patients with a chronic disease, using similar cognitive measures to ours are comparable with our study; the first found that a set of fluid abilities and visual and auditory function completely explained the inverse association between age and TOFHLA-assessed health literacy, ${ }^{18}$ while the second study found that educational differences explained age differences in STOFHLA score. ${ }^{19}$ Other research on this topic has focused on cognitive impairment, rather than a broader range of functional ability such as that captured by the measures in our study. ${ }^{20-23}$ In a previous study, cognitive dysfunction explained the association between increasing age and poorer performance on the TOFHLA and NVS among older adults, while performance on the REALM was best among the oldest adults who screened negative for cognitive dysfunction. ${ }^{23}$ Our study adds the knowledge that subtle individual differences in fluid cognitive ability largely influence age-related differences in the literacy skills required to self-manage health among older adults.

Consistent with evidence showing that speed of visual discrimination, an aspect of processing speed, is 
Figure 1 Health literacy and cognitive abilities by 5-year age group, the LitCog study $(\mathrm{n}=774)$ (NVS, Newest Vital Sign; REALM, Rapid Estimate of Adult Literacy in Medicine; TOFHLA, Test of Functional Health Literacy in Adults).

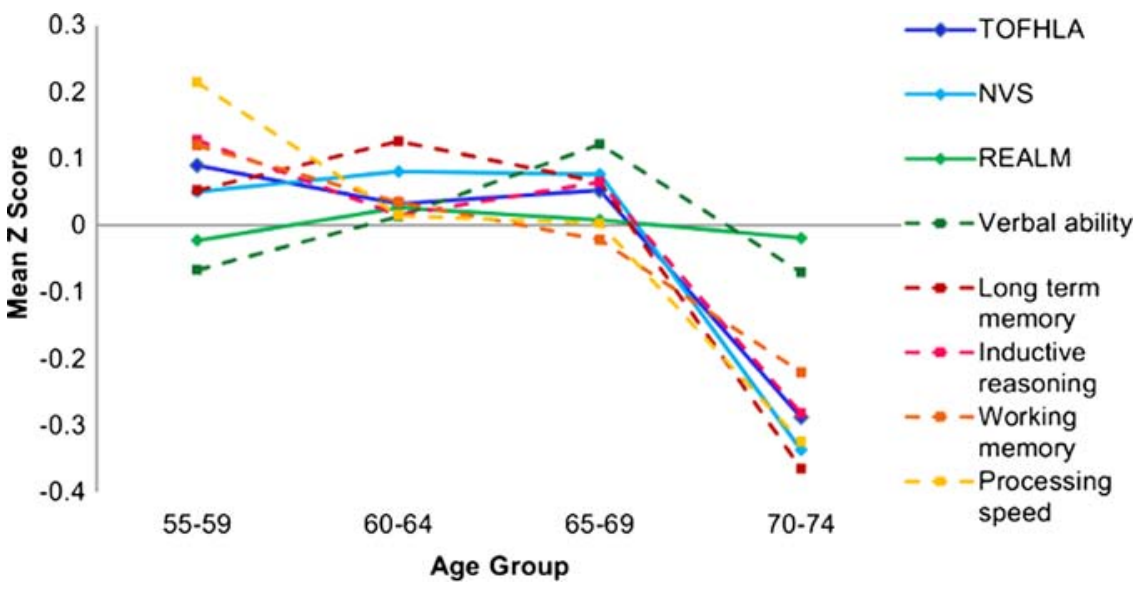

an important marker of cognitive ageing, ${ }^{24-26}$ we found that processing speed was the fluid cognitive ability that individually held the most responsibility for mediating the relationship between age and TOFHLA performance. In LitCog, processing speed was assessed through visual tests of digit and simple line comparisons, and symbol-digit matching. ${ }^{9}$ A similar test of processing speed used in the English Longitudinal Study of Ageing was also shown to mediate the relationship between age and health literacy as assessed by a reading comprehension test. ${ }^{27}$ In the present study, prospective and long-term memory minimally explained age differences in TOFHLA or NVS performance, consistent with previous LitCog research showing that scores on these memory tests are less strongly correlated with TOFHLA and NVS performance than scores on tests of processing speed or inductive reasoning. ${ }^{9}$ These findings provide valuable early insight into the specific fluid abilities that influence performance on health literacy tests and are worth further longitudinal investigation.

As a cross-sectional investigation, we are limited to the extent we can infer a temporal relationship between increasing age and declining health literacy. Health literacy differences between older and younger adults in our study may alternatively be explained by cohort effects, such as by potentially differential educational experiences between generations. As the LitCog study continues, future investigations will consider prospective analyses. Our findings are applicable to English-speaking American adults, and are based on a predominantly female sample. We analysed a comprehensive set of fluid cognitive abilities, but only one measure of crystallised ability. Distributions for each health literacy measure are known to be positively skewed, resulting in ceiling effects. Both the limited crystallised ability measurement and the differing treatment of cognitive and health literacy data may have impacted our findings to a degree, in particular with respect to the threshold and graded trends noted in certain analyses.
Although our study and others indicate that the health literacy skills reliant on fluid cognitive ability are sensitive to age-related decline, longitudinal evidence is needed to elucidate the point at which age-related cognitive decline begins to affect health literacy and, in turn, self-management of health. Whether health literacy decline can be prevented through cognitive-based interventions would be valuable knowledge for the improvement of health autonomy and quality of life for older adults. In particular, improvement of mental processing speed may aid in performance on tests of fluid health literacy skills. Strategies may differ significantly depending on whether health literacy concerns are related to a lack of knowledge and experience in a contextualised healthcare setting, or whether they are related to a lack of the active learning skills that would allow one to access and use new health information. The former scenario would reflect an individual's life experiences and long healthcare use patterns, while the latter scenario could be acquired as a result of a new diagnosis or mild cognitive impairment.

In conclusion, our study indicates that the fluid health literacy skills assessed by the TOFHLA and NVS decline with older age among English-speaking American adults. By contrast, the crystallised health literacy skills assessed by the REALM appear to be stable with age among older adults. Researchers should be mindful of these issues when selecting tests to measure the health literacy of older patients. The ways in which these health literacy tests are constructed result in an operationalisation of health literacy that closely maps onto cognitive abilities that are sensitive to change with age. Clinicians should be aware that health self-management tasks involving comprehension of new information may be increasingly difficult for older patients because of cognitive and literacy burdens. However, performance on health tasks involving the recall of long-term stored knowledge and vocabulary may be relatively unaffected by age. Overall, these results add practical knowledge to help refine the construct of health literacy and its relation to cognitive changes during ageing. 


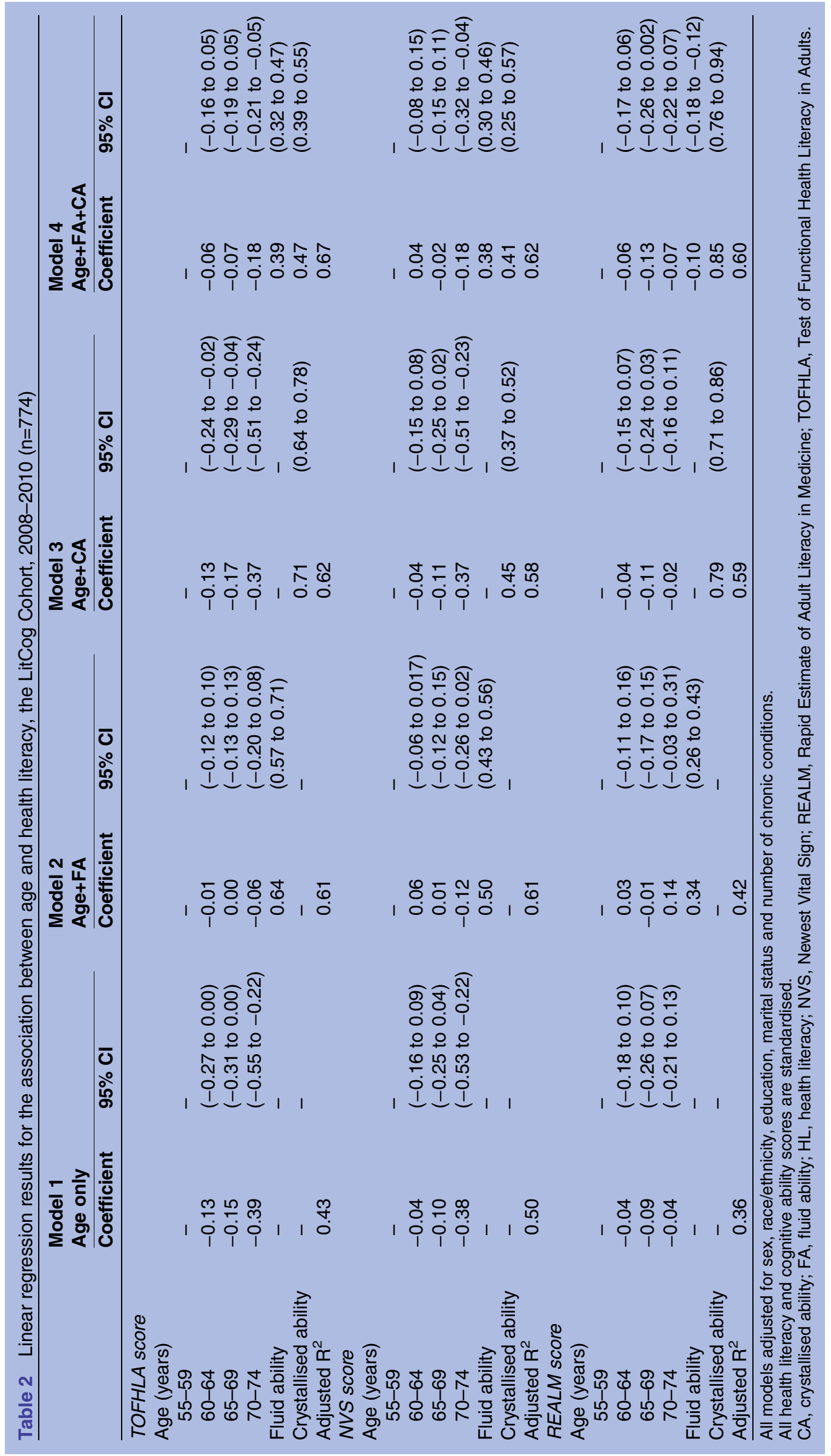




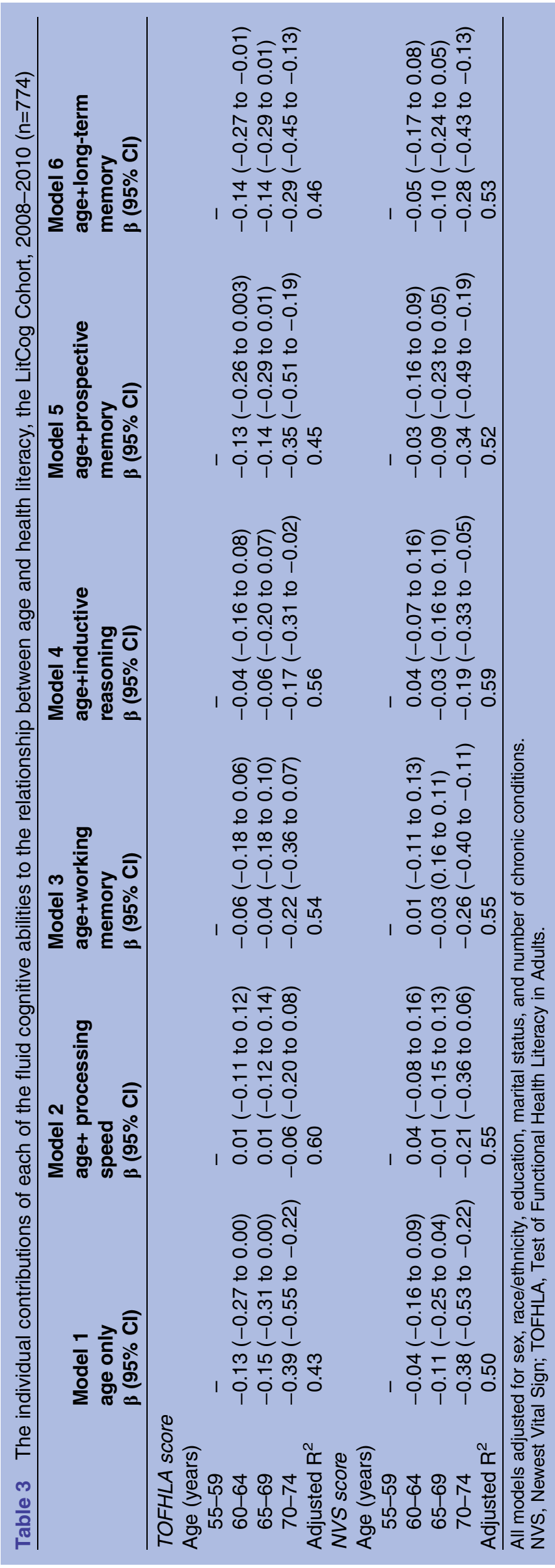

Author affiliations

${ }^{1}$ Department of Epidemiology and Public Health, Health Behaviour Research Centre, University College London, London, UK

${ }^{2}$ Wolfson Institute of Preventive Medicine, Centre for Cancer Prevention, Queen Mary University of London, London, UK

${ }^{3}$ Division of General Internal Medicine, Feinberg School of Medicine at Northwestern University, Chicago, Illinois, USA

${ }^{4}$ Center for Vital Longevity, The University of Texas at Dallas, Dallas,

Texas, USA

${ }^{5}$ Department of Psychology, Centre for Cognitive Ageing and Cognitive Epidemiology, The University of Edinburgh, Edinburgh, UK

${ }^{6}$ Department of Learning Sciences, School of Education and Social Policy, Northwestern University, Evanston, Illinois, USA

Contributors DP, IJD and MSW were responsible for the study concept and design. RO, LMC and MSW were responsible for the acquisition of the data. LCK conducted the statistical analysis and wrote the first draft of the manuscript. All authors contributed to the interpretation of data, critical revision of the manuscript for important intellectual content, and have approved of the final manuscript for publication, and agree to be accountable for all aspects of the work.

Funding The LitCog study is supported by a grant from the National Institute on Aging (R01 AG030611; PI: Wolf).

Competing interests None declared.

Ethics approval Northwestern University's Institutional Review Board.

Provenance and peer review Not commissioned; externally peer reviewed.

Data sharing statement No additional data are available.

Open Access This is an Open Access article distributed in accordance with the Creative Commons Attribution Non Commercial (CC BY-NC 4.0) license, which permits others to distribute, remix, adapt, build upon this work noncommercially, and license their derivative works on different terms, provided the original work is properly cited and the use is non-commercial. See: http:// creativecommons.org/licenses/by-nc/4.0/

\section{REFERENCES}

1. Serper M, Patzer RE, Curtis LM, et al. Health literacy, cognitive ability, and functional health status among older adults. Health Serv Res 2014;49:1249-67.

2. Baker DW, Wolf MS, Feinglass J, et al. Health literacy and mortality among elderly persons. Ann Intern Med 2007;167:1503-9.

3. Kobayashi LC, Wardle J, von Wagner C. Limited health literacy is a barrier to colorectal cancer screening in England: evidence from the English Longitudinal Study of Ageing. Prev Med 2014;61:100-5.

4. Berkman ND, Sheridan SL, Donahue KE, et al. Low Health literacy and health outcomes: an updated systematic review. Ann Intern Med 2011;155:97-107.

5. Kutner M, Greenberg E, Jin Y, et al. The health literacy of America's adults: results from the 2003 National Assessment of Adult Literacy. Washington, DC: National Center for Education Statistics, September 2006; Report No 2006-483. Sponsored by the Department of Education

6. Parker R, Baker D, Williams $M$, et al. The test of functional health literacy in adults: a new instrument for measuring patients' literacy skills. J Gen Intern Med 1995;10:537-41.

7. Weiss B, Mays M, Martz W, et al. Quick assessment of literacy in primary care: the Newest Vital Sign. Ann Fam Med 2005;3:514-22.

8. Davis T, Long S, Jackson R, et al. Rapid assessment of adult literacy in medicine: a shortened screening instrument. Fam Med 1993;25:391-5.

9. Wolf MS, Curtis LM, Wilson EA, et al. Literacy, cognitive function, and health: results of the LitCog study. J Gen Intern Med 2012;27:1300-7.

10. Boyle PA, Yu L, Wilson RS, et al. Cognitive decline impairs financial and health literacy among community-based older persons without dementia. Psychol Aging 2013;28:614-24.

11. Bennett JS, Boyle PA, James BD, et al. Correlates of health and financial literacy in older adults without dementia. BMC Geriatr 2012;12:30

12. O'Carroll R. The assessment of premorbid ability: a critical review. Neurocase 1995;1:83-99. 
13. Salthouse TA. When does age-related cognitive decline begin? Neurobiol Aging 2009;30:507-14.

14. Singh-Manoux A, Kivimaki M, Glymour M, et al. Timing of onset of cognitive decline: results from Whitehall II prospective cohort study. BMJ 2012;344:d7622.

15. Mõttus R, Johnson W, Murray C, et al. Towards understanding the links between health literacy and physical health. Health Psychol 2014;33:164-73.

16. Murray C, Johnson W, Wolf MS, et al. The association between cognitive ability across the lifespan and health literacy in old age: the Lothian Birth Cohort 1936. Intelligence 2011;39:178-87.

17. Kobayashi LC, Wardle J, Wolf MS, et al. Aging and functional health literacy: a systematic review and meta-analysis. $J$ Gerontol $B$ Psychol Sci Soc Sci 2015. Published Online First 11 Dec 2014. doi:10.1093/geronb/gbu161

18. Levinthal BR, Morrow DG, Tu W, et al. Cognition and health literacy in patients with hypertension. $J$ Gen Intern Med 2008;23:1172-6.

19. Morrow D, Clark D, Tu W, et al. Correlates of health literacy in patients with chronic heart failure. Gerontologist 2006: 46:669-76.
20. Armistead-Jehle P, Cifu DX, Wetzel R, et al. Health literacy among patients diagnosed with movement disorders: a pilot study. $P M R$ 2010;2:43-7.

21. Chew LD, Bradley KA, Flum DR, et al. The impact of low health literacy on surgical practice. Am J Surg 2004;188:250-3.

22. Gazmararian JA, Baker DW, Williams MV, et al. Health literacy among Medicare enrollees in a managed care organization. JAMA 1999;281:545-51.

23. Kaphingst KA, Goodman MS, Macmillan WD, et al. Effect of cognitive dysfunction on the relationship between age and health literacy. Pat Educ Couns 2014;95:218-25.

24. Salthouse TA. The processing-speed theory of adult age differences in cognition. Psychol Rev 1996;103:403-28.

25. Deary IJ, Johnson W, Starr JM. Are processing speed tasks biomarkers of aging? Psychol Aging 2010;25:219-28.

26. Ritchie SJ, Tucker-Drob EM, Deary IJ. A strong link between speed of visual discrimination and cognitive ageing. Curr Biol 2014;24:R681-3.

27. Kobayashi LC, Wardle J, Wolf MS, et al. Cognitive function and health literacy decline in a cohort of aging English adults. J Gen Intern Med 2015. Published Online First 14 Feb 2015. doi:10.1007/ s11606-015-3206-9 